INRA Prod. Anim.,

$2013,26(2), 145-156$

\title{
La sélection pour des vaches et une production laitière plus durables : acquis de la génétique et opportunités offertes par la sélection génomique
}

\author{
M. BROCHARD ${ }^{1}$, D. BOICHARD ${ }^{2,3}$, V. DUCROCQ $Q^{2,}$, , S. FRITZ \\ ${ }^{1}$ Institut de l'Elevage, UMR GABI, 149 rue de Bercy, F-75595 Paris, France \\ 2 INRA, UMR1313 GABI, F-78352 Jouy-en-Josas, France \\ 3 AgroParisTech,UMR1313 GABI, F-75231 Paris, France \\ 4 UNCEIA, 149 rue de Bercy, F-75595 Paris, France \\ Courriel : Mickael.Brochard@idele.fr
}

Avec l'arrivée de la sélection génomique et l'évolution des attentes des éleveurs, des citoyens et des consommateurs, l'amélioration génétique des races bovines laitières est à un véritable tournant de son histoire. Quel rôle la génétique joue et jouera-t-elle pour la durabilité de la production laitière?

La croissance des attentes de la société en matière d'environnement est désormais une tendance de fond à laquelle la production laitière n'échappe pas (Cniel 2011). Les conditions de production sont de plus en plus un sujet de débat et l'objet de réglementations « vertes » notamment au niveau européen : limitation des intrants, gaz à effet de serre, rejets azotés, promotion du bio, bien-être animal, etc. Le syndrome métabolique devient un problème majeur de santé publique, les inquiétudes d'une partie des consommateurs vis-à-vis de la qualité nutritionnelle des produits laitiers notamment, en font un des principaux enjeux des filières laitières de demain. En parallèle, les éleveurs sont de plus en plus en attente d'animaux « fonctionnels » ou faciles à élever pour réduire les coûts, interventions et astreintes dans des élevages de taille croissante. Est-ce que la sélection apporte et apportera demain des éléments de réponse à ces nouvelles attentes ? Estce qu'elle saura produire les vaches dont l'agriculture écologiquement intensive a besoin ? Quelle adaptation aux conditions d'élevage de demain et à leur diversité ? Sera-t-elle un des acteurs essentiels de l'agriculture durable?

La durabilité des animaux et des systèmes de production est une notion qui comprend plusieurs aspects. En matière de sélection, les facteurs principaux de durabilité sont : la robustesse, l'efficacité alimentaire et la limitation des rejets, ainsi que la qualité sanitaire, nutritionnelle et technologique du lait. La robus- tesse des animaux (Bodin et al 2010) est ici considérée comme l'ensemble des aptitudes dites fonctionnelles : ensemble de caractères relatifs à la capacité d'un animal à vivre, se reproduire et produire en bonne santé dans un contexte d'élevage donné, avec le moins possible d'interventions de l'Homme. On parle également de qualité ou facilité d'élevage. Cela concerne des aptitudes telles que la fertilité, la résistance aux maladies, la longévité des animaux, etc. La notion de qualité globale du lait produit par ces animaux recouvre aussi différents champs : sanitaire, nutritionnel et technologique.

Les développements scientifiques et techniques des dernières décennies ont permis d'améliorer la maîtrise et l'optimisation des différentes étapes des programmes de sélection, les rendant ainsi très performants. La compétitivité des exploitations laitières françaises s'est alors accrue, essentiellement par une augmentation substantielle de la productivité laitière, accompagnée d'un accroissement de la richesse du lait en matières protéiques (Verrier et al 2010). D'abord absents des objectifs de sélection en dehors de la morphologie, les caractères fonctionnels y ont été intégrés progressivement depuis la fin des années 1990.

Aujourd'hui, avec la sélection génomique, les leviers génétiques sont profondément renouvelés, à tel point que certains parlent de « révolution ». Cette innovation est utilisée dès à présent pour augmenter l'efficacité de la sélection sur la robustesse des animaux en particulier. C'est également une formidable opportunité pour prendre en compte toute une série de nouveaux caractères en réponse aux besoins liés à la durabilité, notamment dans les domaines de la santé animale, de l'efficacité alimentaire ou encore de la composition fine du lait. L'accès aux performances des animaux, dites aussi " phénotypes », devient alors un enjeu majeur. De nombreux programmes de phénotypage sont mis en œuvre, modifiant profondément les relations entre les acteurs, les outils et les méthodes d'approche des caractères.

\section{1 / 50 ans de sélection géné- tique : résultats et limites par rapport à la durabilité}

Dans la plupart des pays développés, au cours des 30 dernières années, le progrès génétique en production laitière variait entre 50 et $100 \mathrm{~kg}$ par lactation chaque année. Toutes les races ont été concernées, hormis celles dont les effectifs limités d'animaux n'ont pas permis la mise en œuvre de programmes de sélection mais justifiaient des mesures conservatrices. La composition du lait a généralement été un caractère secondaire et son évolution a été assez faible, en augmentation pour le taux protéique et plus variable selon les conditions économiques du moment pour le taux butyreux. Compte tenu de la corrélation génétique négative presque systématique 
entre la productivité laitière et les caractères fonctionnels - la fertilité, la résistance aux mammites et à d'autres maladies, la longévité -, la robustesse générale des vaches laitières s'est dégradée dans le même temps (Jorjani et al 2007). Les caractères fonctionnels sont difficiles à sélectionner de manière classique à cause de leur héritabilité modérée à très faible (par exemple, l'héritabilité du taux de conception est comprise entre 1 et $3 \%$ ), mais leur variabilité génétique est importante, ce qui les rend susceptibles d'être rapidement détériorés en réponse indirecte à une sélection fortement orientée vers la productivité, par exemple le taux de réussite à l'insémination a baissé de près d' 1 point entre 1998 et 2001 du fait de la dégradation des aptitudes génétiques (Le Mézec et al 2010). En parallèle, la réduction des coûts et des interventions est devenue l'objectif principal de la conduite de troupeaux de taille croissante, avec moins de main-d'œuvre. En Amérique du Nord (Rogers 2005) comme en Europe, certains éleveurs privilégient même le croisement ou un changement de race pour contourner rapidement les effets indésirables de cette dégradation de la robustesse des vaches.

Depuis le début des années 2000, la majorité des programmes de sélection intègrent les caractères fonctionnels, suivant l'exemple pionnier des pays scandinaves. En France, grâce à un fort investissement méthodologique de l'INRA, les éleveurs des différentes races laitières ont pu disposer progressivement d'évaluations génétiques pour différents caractères de robustesse des vaches : en 1997, les cellules somatiques (Rupp et Boichard 1997) et la longévité fonctionnelle (Ducrocq 2005) ; en 1998, le taux de conception chez la vache (Boichard et al 1998); en 2010, les occurrences de mammites cliniques ainsi que d'autres caractères de fertilité (taux de conception pour les génisses et intervalle vêlage- $1^{\text {ère }}$ insémination). Notons également les évaluations génétiques sur les conditions de naissance et de vêlage disponibles depuis 2000 et la viabilité des veaux à la naissance depuis 2007 .

\section{1 / Caractères de robustesse}

\section{a) Fertilité des femelles}

Il s'agit en fait d'un ensemble de caractères (aptitude à concevoir, aptitude à la reprise de cyclicité) dont l'évaluation requiert un recueil national de l'ensemble des inséminations (facteur limitant dans de nombreux pays) mais aussi des vêlages et des états de présence et de lactation. En France, nous utilisons le taux de conception ou taux de réussite après chaque insémination pour les génisses et les vaches, ainsi que l'inter- valle vêlage- $1^{\text {ere }}$ insémination, servant d'indicateur de la reprise de cyclicité.

\section{b) Résistance aux mammites}

À l'exception notable des pays nordiques qui disposent depuis longtemps d'un système remarquable de recueil des données de santé, la résistance aux mammites a été et est encore très majoritairement abordée indirectement par la concentration en cellules somatiques dans le lait. Outre sa relative simplicité, cette mesure présente l'avantage de bien prendre en compte les mammites subcliniques persistantes. En revanche, elle n'explique que partiellement les mammites cliniques : la corrélation génétique entre comptages cellulaires et fréquence de mammites cliniques est comprise entre 0,55 et 0,73 (Rupp et Boichard 1999, Bonaïti et al 2005). Un système d'enregistrement des mammites cliniques a été mis en place (Douguet et al 2008) et permet à la France d'être parmi les rares pays à disposer d'une évaluation génétique de la résistance aux mammites cliniques.

c) La longévité fonctionnelle ou durée de vie productive (du $1^{\text {er }}$ vêlage à la réforme)

Il s'agit d'un caractère avec un statut particulier : il peut être vu comme un indicateur global de robustesse, résultant de défaillances sur d'autres caractères. On parle de longévité « fonctionnelle», c'est-à-dire l'aptitude à prévenir une réforme involontaire (fécondité, problème de santé, boiterie...), par opposition aux réformes volontaires (principalement dues à une production laitière jugée insuffisante).

\section{d) Caractères morphologiques}

Depuis la création des races, une forte sélection sur certains caractères morphologiques des vaches (taille, apparence laitière...) a été mise en avant par bon nombre d'éleveurs et de vendeurs de reproducteurs ou de semences comme un moyen d'accroître la longévité des vaches (Larroque et Ducrocq 2001). De nombreux travaux ont ébranlé cette affirmation et au contraire certaines pratiques de sélection sur certains de ces caractères ont eu des répercussions fâcheuses sur la robustesse des animaux. Par exemple, la liaison entre la robustesse et la taille des vaches est controversée, voire s'est avérée clairement défavorable dans certains travaux (Pryce et al 2009). De même, en race Holstein, la sélection sur l'apparence laitière («dairy form ») a des conséquences néfastes sur la fertilité et la longévité (Lassen et al 2003). A l'opposé, on retrouve dans toutes les études un impact favorable de la sélection de caractères liés à la morphologie de la mamelle sur la résistance aux mammites, la vitesse de traite et la longévité, et sur la commodité du travail. La situation est moins claire en ce qui concerne les caractères liés à la morphologie des aplombs : peu héritables, ils sont finalement faiblement liés à l'incidence des boiteries (Uggla 2008, Stoop et al 2010).

\section{2 / Evaluations génétiques natio- nales et internationales}

L'évaluation génétique de certains caractères fonctionnels nécessite un traitement statistique particulier, à cause de spécificités incompatibles avec les hypothèses classiques, comme par exemple la nature discontinue des données ou la variabilité de l'échelle de mesure subjective (ex. : conditions de naissance). La situation la plus extrême est la longévité fonctionnelle $: i)$ les données sont dites censurées (les plus jeunes animaux - ceux qui nous intéressent en sélection ne sont pas encore réformés !) ; ii) les facteurs de milieu influençant les choix de réforme changent en même temps que ce que l'on mesure (la durée de vie); iii) le caractère est affecté par les réformes volontaires liées à une production laitière insuffisante (Ducrocq 2006).

Au plan international, bien que la plupart des caractères fonctionnels soient intégrés dans les évaluations génétiques internationales réalisées par l'organisme Interbull (www.interbull.org, Banos 2010), l'amélioration de la robustesse par les échanges de reproducteurs et de semences entre pays n'est pas optimale. Tout d'abord, l'importance accordée aux caractères de morphologie lors du choix des reproducteurs reste généralement excessive (supérieure à l'importance accordée aux autres caractères fonctionnels). Ensuite, du fait d'une grande hétérogénéité de définition des caractères fonctionnels, des systèmes de collecte et des modèles d'évaluation génétique, les corrélations génétiques entre différentes mesures du même caractère dans différents pays sont faibles et empêchent souvent les meilleurs taureaux étrangers d'apparaître dans les « top lists » nationales.

\section{3 / Objectifs de sélection et index de synthèse}

La mise en place d'évaluations génétiques sur la fertilité, la longévité ou les cellules somatiques n'a eu aucun impact tant que ces caractères n'étaient pas inclus dans des index synthétiques correspondant aux objectifs globaux de sélection.

Jusqu'à la fin des années 1990, les objectifs de sélection des pays laitiers combinaient essentiellement les caractères laitiers (production de matière 
protéique et de matière grasse, taux de matière protéique et de matière grasse du lait) et des caractères de morphologie. Avec l'urgence de stopper la dégradation des caractères fonctionnels, ces objectifs de sélection se sont considérablement élargis et équilibrés (Colleau et Regaldo 2001), avec un poids relatif décroissant des caractères laitiers. Depuis février 2012, l'objectif de sélection de la race Prim'Holstein en France accorde un poids prépondérant aux caractères non liés directement à la production : $22 \%$ à la fertilité, $18 \%$ à la santé de la mamelle, $15 \%$ à la morphologie, $5 \%$ à la longévité, $5 \%$ à la vitesse de traite, contre $35 \%$ à la production qui devient minoritaire.

L’index synthétique (ou ISU pour «Index Synthétique Upra») combine les évaluations génétiques pour les différents caractères qui constituent l'objectif de sélection. Or, les valeurs génétiques sur les caractères fonctionnels sont souvent disponibles seulement pour les taureaux, avec initialement de faibles valeurs du Coefficient de Détermination (CD), qui est un indicateur de la précision de l'évaluation. Souvent, des prédicteurs précoces sont disponibles pour accroître cette précision (ex. : morphologie de la mamelle pour la résistance aux mammites). Contrairement à la plupart des pays qui utilisent de simples combinaisons linéaires d'index, la France a développé et mis en œuvre depuis 2001 une évaluation multicaractère approchée
(Ducrocq et al 2001, Lassen et al 2007). Cette méthode permet de contourner les difficultés, en combinant de façon optimale toutes les informations disponibles en fonction des corrélations génétiques et des différences de précision d'un caractère à l'autre.

Le virage pris par les programmes de sélection, particulièrement avec la réforme de l'ISU en 2001, se traduit, comme le montre l'examen des courbes de progrès génétique (figure 1) sur le taux de conception des vaches et les comptages de cellules somatiques, par un arrêt de la dégradation génétique voire une légère amélioration de ces caractères à partir des générations de femelles issues de taureaux disposant d'un ISU (Dezetter et Le Mézec 2011). Comme nous le verrons plus loin, la mise en œuvre de la sélection génomique devrait permettre une amélioration beaucoup plus nette.

\section{2 / Les leviers génétiques renouvelés avec la sélection génomique}

\section{1 / Principe général des évalua- tions génomiques}

Suite au séquençage du génome bovin, des outils de génotypage à grande échelle ont été développés. Ces outils sont dési- gnés sous le terme de « puces », du fait de leur miniaturisation. La puce « BovineSNP50 » fournie par la firme «Illumina », utilisée en France depuis 2008, permet de suivre la transmission des régions chromosomiques au cours des générations (Robert-Granié et al 2011). Les évaluations ou indexations génomiques utilisent alors trois types d'informations : généalogies enregistrées, performances mesurées et génotypes observés. La méthode générale repose sur la constitution d'une population de référence suffisamment grande, constituée d'individus pour lesquels on dispose à la fois des phénotypes d'intérêt et des génotypes. Le génotype des individus est obtenu par la détermination des bases nucléotidiques ( $\mathrm{A}, \mathrm{T}, \mathrm{G}$ ou $\mathrm{C}$ ) d'une série de marqueurs répartis sur le génome. Cette population permet d'étudier les relations entre phénotypes et marqueurs et de construire un outil de prédiction de la valeur génétique. L'outil ainsi développé peut être utilisé pour prédire la valeur génétique d'un (jeune) animal sur la base de son seul génotype aux marqueurs, bien avant de connaître ses performances individuelles, ou sur descendance. La précision des index génomiques calculés dépend de la taille de la population de référence et de l'héritabilité du caractère (Hayes et al 2009). En France, en janvier 2012, les populations de référence des races Prim'Holstein, Montbéliarde et Normande sont constituées respectivement de 22000,1800

Figure 1. Progrès génétiques des taureaux Prim'Holstein pour les caractères composants l'ISU (Dezetter et Le Mézec 2011).

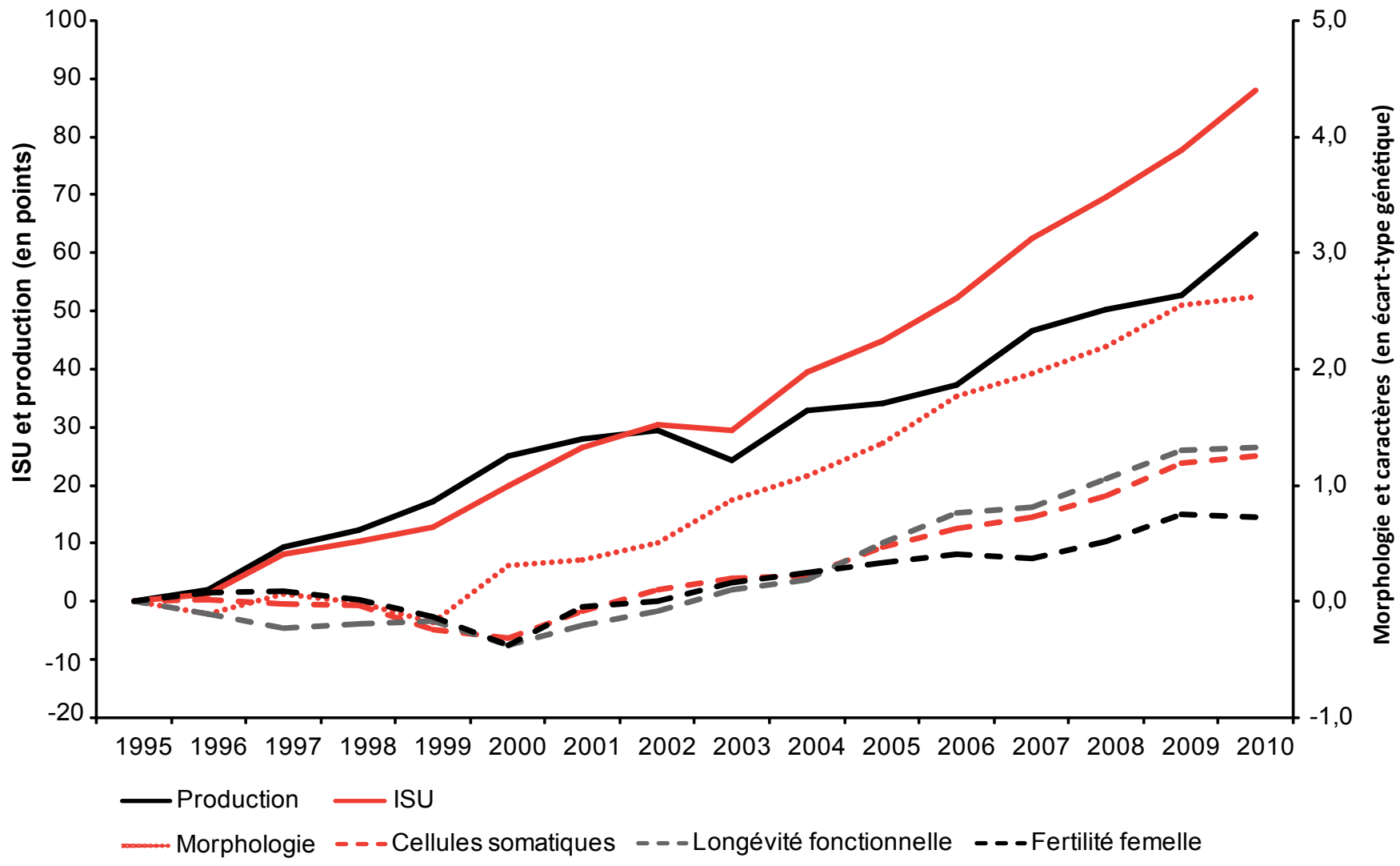


et 1500 taureaux génotypés sur la puce « BovineSNP50 » et également testés sur descendance, ce qui représente des millions de phénotypes collectés par caractère dans ces trois races. Grâce à ces populations de référence et aux travaux réalisés dans le cadre du projet Approches Méthodologiques et Application de la Sélection GENomique (AMASGEN), financé par l'Agence Nationale pour la Recherche (ANR) et le fond de recherche Apis-Gène (www.apis-gene.fr), les jeunes animaux disposent d'estimations de leurs valeurs génétiques avec un $\mathrm{CD}$ supérieur à 0,50 pour 1 'ensemble des caractères sélectionnés (tableau 1). Cette valeur de 0,50 de CD est très importante à considérer puisqu'elle représente, pour les caractères laitiers, le seuil au-delà duquel il est, selon la réglementation européenne, possible d'utiliser le taureau en insémination.

Tableau 1. Coefficients de Détermination (CD) moyens des index génomiques calculés en juin 2010 (Fritz et al 2010).

\begin{tabular}{|l|c|c|c|}
\hline Race & LAIT & CEL $^{(1)}$ & FERv $^{(2)}$ \\
\hline Prim'Holstein & 0,70 & 0,67 & 0,60 \\
\hline Montbéliarde & 0,64 & 0,62 & 0,53 \\
\hline Normande & 0,62 & 0,61 & 0,51 \\
\hline
\end{tabular}

\footnotetext{
(1) score de cellules somatiques.

(2) fertilité femelle des vaches.
}

\section{2 / Conséquences pour les pro- grammes de sélection en bovins laitiers}

L'utilisation des index génomiques dans les programmes de sélection est appelée sélection génomique. L'expression " révolution génomique " assume le fait que cet outil permet une évaluation génétique très précoce de la vie d'un reproducteur, voire au stade embryonnaire. Plusieurs scénarios s'appuyant sur la sélection génomique ont été testés par simulation sur une grande population de bovins laitiers, sélectionnée pendant 20 ans sur un index de synthèse mimant l'ISU (Colleau et al 2009). D'après cette simulation (figure 2), les scénarios sans étape de testage sur descendance (AXMAX et AXMIX) conduisent tous deux à une augmentation de +84 et $+88 \%$ du progrès génétique annuel par rapport au scénario REF (présélection sur index génomiques, puis testage des candidats sur descendance), en accord avec les premiers résultats de Schaeffer (2006). Ces deux scénarios diffèrent en matière de durabilité car AXMAX (les taureaux ne sont utilisés que durant une seule campagne d'insémination et chacun d'entre eux est père à taureau) est nettement plus favo-

Figure 2. Progrès génétique annuel et évolution de consanguinité selon trois scénarios de sélection génomique (Colleau et al 2009).

REF, AXMAX et AXMIX sont trois scénarios de sélection génomique sur une grande population de bovins laitiers appliqués pendant 20 ans. Le scénario REF consiste en une présélection des candidats puis testage sur descendance. Les deux autres scénarios se distinguent de REF par la suppression de cette étape de testage sur descendance AXMAX considère que les taureaux, même les meilleurs, ne sont utilisés pendant une seule campagne et sont tous pères à taureaux alors que AXMIX considère la réutilisation des meilleurs taureaux.

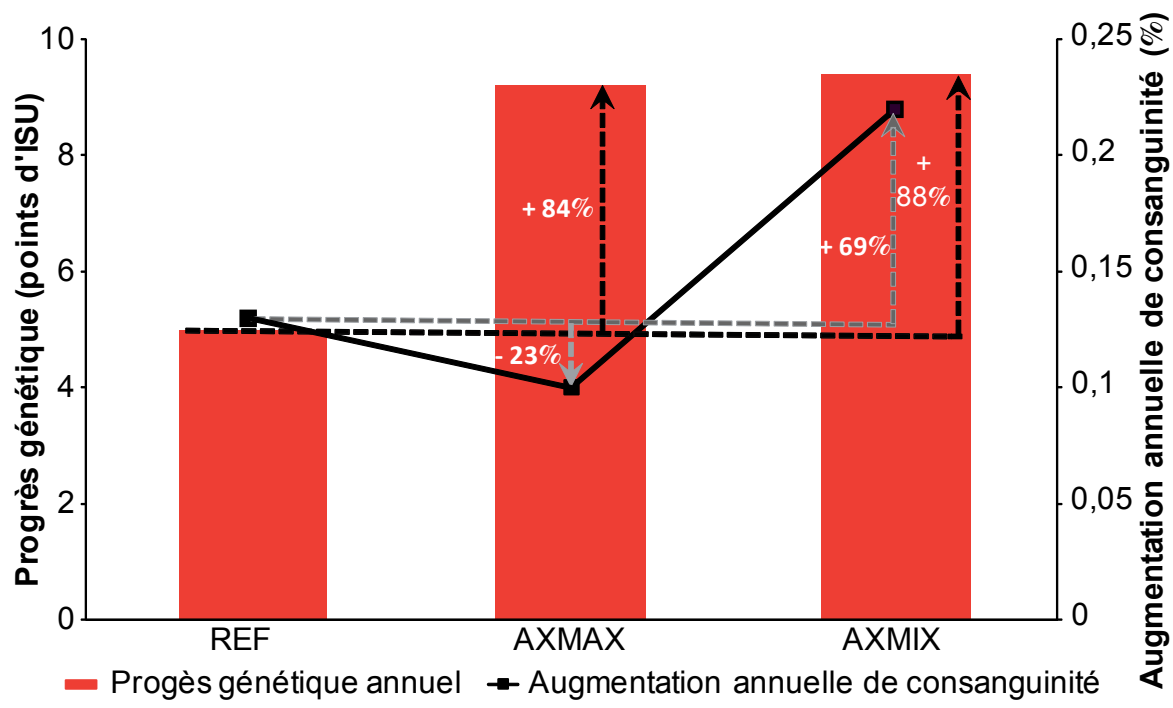

rable à la variabilité génétique qu'AXMIX (les meilleurs taureaux sont utilisés 2 ans et surtout les meilleurs d'entre eux une fois l'information sur descendance obtenue, sont réutilisés et réalisent $50 \%$ des inséminations) : - $23 \%$ d'évolution annuelle de consanguinité pour AXMAX par rapport à REF, contre $+69 \%$ pour AXMIX.

Ces résultats montrent qu'en sélection génomique chez les bovins laitiers, il est parfaitement possible de quasiment doubler le progrès génétique sans conséquence néfaste pour l'évolution annuelle de consanguinité, grâce à l'utilisation équilibrée d'un plus grand nombre de jeunes reproducteurs, diffusés sur la base de leurs index génomiques sans période de testage sur descendance. Compte tenu du niveau légèrement plus faible de précision des index génomiques des jeunes taureaux sans descendance, il est toutefois conseillé de raisonner par groupes de taureaux afin de limiter les risques et garantir ce progrès génétique élevé.

\section{3 / Opportunités offertes par la sélection génomique}

\section{a) Un outil à disposition des éleveurs}

Afin de rendre la sélection génomique accessible aux éleveurs à un coût le plus faible possible, une nouvelle puce dite «BovineLD » a été développée par Illumina en 2011, dans le cadre d'un consortium international (Boichard et al 2012). Cette nouvelle puce ne compte que 6909 marqueurs SNP, en grande majorité aussi présents sur la puce BovineSNP50. Les marqueurs ont été choisis de façon à optimiser les travaux d'imputation. L'imputation consiste à prédire les typages manquants d'un animal et conduit à disposer de tous les SNP de la puce BovineSNP50 à partir des 6909 SNP réellement génotypés (Dassonneville et al 2012). La qualité de l'imputation dépend des populations de référence réellement typées sur la puce BovineSNP50. Cet outil vise en particulier l'indexation génomique en élevage à très grande échelle des populations femelles.

b) La voie femelle, un nouveau levier pour les caractères fonctionnels

La sélection génomique permet d'avoir des index aussi précis pour les jeunes femelles que pour les jeunes mâles et ce même pour les caractères faiblement héritables que sont les caractères fonctionnels (tableau 1). L'évaluation classique, faute de CD suffisant, ne permettait pas de disposer pour les femelles d'index relatifs aux caractères fonctionnels (en dehors des comptages cellulaires). L'indexation génomique en élevage devient un outil de gestion du troupeau femelle.

\section{c) Enrichir les objectifs de sélection:} vers plus d'équilibre

Dans un second temps, la sélection génomique doit permettre d'intégrer de façon efficace de nouveaux caractères dans les objectifs de sélection. D'une 
part, comme elle augmente le progrès potentiel, elle donne l'opportunité d'augmenter le poids des caractères de robustesse (cf. 1.3, le nouvel ISU adopté depuis 2012) et d'intégrer de nouveaux caractères aux objectifs de sélection actuels, sans pénaliser le progrès laitier. D'autre part, elle déconnecte contrôle de performances et sélection, et permet d'envisager de collecter de nouveaux phénotypes dans une population de référence (cf. § 4.1) éventuellement adaptée à chaque caractère. A titre d'exemple nous pouvons citer le programme PhénoFinlait (www.phenofinlait.fr, Esvan et al 2010) sur la composition fine du lait ou encore le projet PARADIGM sur la paratuberculose.

\section{3 / Nouveaux caractères, opportunités pour la dura- bilité ?}

Au cours du temps, les objectifs de sélection des bovins laitiers ont intégré un nombre croissant de caractères et sont devenus de plus en plus élaborés. Ce phénomène est général et a été observé dans la plupart des espèces d'élevage : la sélection vise tout d'abord à améliorer les caractères qui sont le plus limitant, puis elle prend progressivement en compte d'autres caractères, en fonction des besoins. Par ailleurs, au départ, elle doit être très efficace et peu coûteuse, ce qui est permis si on se limite à un objectif simple. La complexification nécessite davantage de mesures sur les animaux, donc une organisation plus coûteuse. Elle entraîne une « dilution » de l'effort de sélection au fur et à mesure que le nombre de caractères s'accroît et donc un progrès plus faible sur chacun d'eux. Elle n'est donc possible que lorsque le système est mature. Au cours des 50 dernières années, les objectifs de sélection ont progressivement pris en compte la productivité laitière, la richesse du lait, la morphologie, et les caractères fonctionnels, de plus en plus nombreux (Verrier et al 2010). Bien que déjà très complet, l'objectif actuel ne couvre pas toutes les composantes souhaitables ou envisageables, principalement pour des raisons de difficulté de collecte de l'information phénotypique correspondante et, dans une moindre mesure, à cause de la faible héritabilité, limitant l'efficacité de la sélection.

On peut supposer qu'à l'avenir les objectifs de sélection continueront à évoluer vers plus de complexité, et ce pour plusieurs raisons : des possibilités d'accès à de nouveaux phénotypes grâce à une meilleure intégration de l'information ou la disponibilité de nouvelles technologies de mesure; des besoins quantitatifs en phénotypes plus réduits pour la sélection génomique que pour la sélection classique ; des besoins nouveaux, liés à des demandes des filières, des consommateurs ou de la société. Trois grands domaines peuvent être explorés : la robustesse et ses différentes composantes ; l'efficacité de la production et la limitation de l'impact environnemental; la qualité, en particulier nutritionnelle, des produits.

\section{1 / Robustesse des animaux}

L'amélioration génétique doit faire face à un nouveau défi qui est le transfert de « la maîtrise des effets de l'environnement par le système de production à leur maîtrise par l'animal lui-même " (Bodin et al 2010). Ce transfert est nécessité notamment par la «mise en place d'une agriculture durable [qui] doit mobiliser des systèmes de production dans lesquels le milieu est moins contraint et contrôlé » (Bodin et al 2010). Parmi les composantes du moindre « contrôle » du milieu, il y a par exemple la réduction de la disponibilité des éleveurs pour le suivi individuel des animaux (connue depuis plusieurs décennies en lien avec l'augmentation de la taille des structures de production par unité de main-d'œuvre), la réduction des intrants notamment médicamenteux, etc. Dans ce contexte, la robustesse en général - au sens de la capacité d'un animal à vivre, se reproduire et produire en bonne santé dans un contexte d'élevage avec le moins possible d'interventions de l'Homme - et ses différentes composantes (santé, reproduction, longévité, adaptation au système de production...) constituent un enjeu considérable.

La santé est sans doute le point le moins pris en compte jusqu'à ce jour, limité pour l'instant à la résistance aux mammites. Une voie importante d'amélioration consiste à mieux utiliser les données sanitaires présentes dans les élevages, concernant par exemple les maladies métaboliques, digestives, respiratoires, reproductives, etc. L'exemple nous est donné à l'étranger, bien sûr dans les pays nordiques, et plus récemment au Canada et en Autriche (EggerDanner et al 2010). Les données existant déjà, cette action nécessite surtout une normalisation des informations, avec une standardisation de leur enregistrement et leur gestion dans une base unique. Les troubles des pieds et des sabots constituent une cause importante de réforme. Le projet PARABOV (financé par France Génétique Elevage, interprofession des acteurs de l'amélioration génétique des ruminants d'élevage) cible les données de pathologie des pieds, obtenues par les pareurs dans le cadre de leur activité. Avant toute utilisation, il convient de les standardiser et de les rassembler. A nouveau, des exemples internationaux existent, par exemple en Suède, au Canada ou aux Pays-Bas (Eriksson 2006, Cramer et al 2008, Stoop et al 2010). La susceptibilité à la paratuberculose fait également l'objet de suivis mais, contrairement aux cas précédents, les données n'existent pas et leur production est particulièrement exigeante. Elle peut reposer sur un suivi longitudinal de troupeaux affectés ou, de façon moins précise, sur les diagnostics à l'abattage. Différentes initiatives à l'étranger montrent cependant que cette action est possible (Gonda et al 2006, Minozzi et al 2010).

L'état corporel est un autre caractère très important. Il est associé à la mobilisation corporelle, une composante essentielle de la physiologie de la vache laitière, mais aussi à l'état de santé, la capacité de reproduction et à la longévité. Sa mesure est toujours complexe et au-delà des notes d'état, en partie subjectives, on peut envisager divers dispositifs $: i)$ la mobilisation étant associée à la composition de la matière grasse du lait (d'autres bio-marqueurs comme des métabolites ou des hormones pourraient être considérés également), une mesure peut être basée sur la composition fine du lait (McParland et al 2011); ii) des pesées répétées plusieurs fois par jour à l'aide de dispositifs automatiques permettent sans doute d'estimer correctement l'évolution du poids et le bilan énergétique sur le court terme (Friggens et al 2011); iii) enfin, des études montrent que l'imagerie externe permet d'appréhender la forme de l'animal et donc son angularité (Bewley et al 2008, Halachmi et al 2008).

Les races bovines laitières étant des populations de petite taille génétique (Danchin-Burge et al 2012), leur taux de consanguinité augmente régulièrement et s'accompagne notamment de l'accroissement de l'incidence des tares génétiques récessives. A partir de quelques cas, les méthodes actuelles fondées sur le génotypage et le séquençage à haut débit permettent de localiser l'anomalie, souvent d'identifier la mutation causale, et toujours de disposer de tests de diagnostic permettant d'identifier les porteurs hétérozygotes. Le facteur limitant reste la détection précoce des cas d'accroissement d'incidence et nécessite la mise en place d'observatoires spécialisés comme au Danemark (Agerholm 2007) ou en France (www. onab.fr). Plus récemment, l'analyse des données de génotypage à haut débit des populations en sélection génomique a permis de mettre en évidence plusieurs anomalies de développement conduisant à des mortalités embryonnaires ou fœtales (VanRaden et al 2011), induisant une baisse de fertilité. 
D'autres phénotypes pourront sans doute être appréhendés dans le futur : à partir de capteurs en ferme variés comme les accéléromètres pour l'analyse du comportement, la détection des chaleurs et de différents troubles de santé (boiteries, position couchée prolongée...) ; l'analyse du lait pour la détection de troubles divers de l'animal ; le bolus ruminal pour l'analyse de la température et donc la détection de pathologies ; le comportement alimentaire etc. La gamme est quasi infinie, mais il convient de définir précisément le diagnostic d'intérêt ainsi que les conditions d'accès aux données, pour permettre de normaliser, collecter, stocker et gérer des informations pertinentes et valorisables à des fins de sélection.

\section{2 / Efficacité alimentaire et rejets}

L'alimentation représente le coût de production le plus important. L'efficacité alimentaire a été améliorée chez la vache laitière essentiellement par augmentation de la productivité et réduction concomitante de la part de l'entretien dans les besoins alimentaires. Mais peu de choses sont connues sur l'efficacité digestive et les pertes ruminales, deux composantes potentiellement importantes de l'efficacité alimentaire. La principale difficulté réside dans la mesure du caractère. Il semble qu'efficacité alimentaire et rejet de méthane soient corrélés, une perte de méthane induisant une perte d'énergie (Hegarty et al 2007). La mesure de l'ingestion de la ration est coûteuse en équipement et en travail, ce qui en limite grandement sa mise en œuvre à grande échelle. De même, les dispositifs de mesure de la production de méthane sont souvent très lourds et peu envisageables en dehors de structures expérimentales et sur des effectifs relativement réduits (PinaresPatiño et al 2003). Toutefois, un dispositif simplifié a été mis en place au Danemark (Lassen et al 2010) pour mesurer la production de méthane, en comparaison de celle du gaz carbonique, par spectrométrie Moyen Infra Rouge (MIR) au moment de la traite dans un robot légèrement confiné. La mesure est peu précise mais elle peut être répétée à chaque traite, fournissant alors une bonne précision par animal. D'autres travaux, parmi lesquels ceux de Chilliard et al (2009) ont établi des relations entre composition du lait en acides gras et rejet de méthane. Cette utilisation de biomarqueurs dans le lait laisse entrevoir des capacités de phénotypage à grande échelle mais elle requière de connaître l'alimentation des animaux.

Ces mesures resteront complexes et des collaborations larges et internationales (par exemple le partenariat gDMI : "global Dry Matter Initiative", Veerkamp et al 2012) se mettent en place pour constituer des populations de référence suffisantes sur ce caractère difficile à prendre en compte mais important.

\section{3 / Composition fine du lait}

La composition fine du lait fait l'objet de nombreuses études depuis les travaux pionniers de la Faculté Universitaire des Sciences Agronomiques de Gembloux (Soyeurt et al 2006). Le principe est simple : la spectrométrie dans le MIR, utilisée pour prédire les taux butyreux et protéique, peut aussi être utilisée pour doser d'autres composants. Des équations de prédiction peuvent être développées à partir d'échantillons caractérisés à la fois avec une mesure de référence et un spectre MIR. En disposant d'équations de prédiction, on valorise ainsi les millions de spectres MIR produits chaque année dans le cadre du contrôle laitier. Les travaux récents (Soyeurt et al 2006, Ferrand et al 2011) montrent que l'on peut caractériser avec une précision correcte une vingtaine d'acides gras majeurs ainsi que divers ratios comme le taux de désaturation (Soyeurt et al 2009a), mais aussi certaines protéines (avec une précision moindre cependant), la lactoferrine (Soyeurt et al 2007), les minéraux (Soyeurt et al 2009b) ou les propriétés de coagulation (Dal Zotto et al 2008, Cecchinato et al 2009) ou d'aptitude fromagère des laits (De Marchi et al 2009). La méthode est également utilisable pour caractériser l'animal, dès lors que son état (mobilisation corporelle ou santé par exemple) se traduit par une modification détectable et interprétable de la composition du lait.

L'approche est très attractive et en partie à l'origine du programme français d'étude de la composition fine du lait, PhénoFinlait (www.phenofinlait.fr, Esvan et al 2010). Ce programme multidisciplinaire, associant les différentes composantes des filières laitières bovine, caprine et ovine et les acteurs de la recherche et du développement, vise à mesurer à grande échelle (plus de 1500 élevages suivis pendant 1 an) la composition fine (acides gras et protéines) des laits des principales populations laitières françaises. Parallèlement à ce phénotypage une population de femelles a été génotypée, ce qui devrait donner lieu rapidement à des index sur la composition fine du lait, au moins pour le profil en acides gras. Plusieurs problèmes restent cependant à résoudre. Tout d'abord, comme pour tous les phénotypes, il convient de stocker les données des spectres MIR. Compte tenu de leur nombre et de leur volume (plus de 1000 points (x, y) par spectre), il s'agit d'un premier exemple pratique de phénoty- page à haut débit. Par ailleurs, les spectromètres nécessitent une calibration pour fournir des données interprétables sans biais, un problème qui n'est pas encore réglé de façon simple autrement que par l'analyse régulière de laits témoins.

\section{4 / Où il est question de phé- notypage}

Avec la sélection génomique est apparue la notion de population de référence : un nombre plus ou moins grand d'animaux à partir desquels sont établies des correspondances entre génotypes à une série de marqueurs et phénotypes. Ces correspondances ou équations sont ensuite extrapolées aux candidats sans phénotypes. La question de l'accès à des phénotypes et à des populations de référence de qualité est alors absolument stratégique.

\section{1 / Phénotypage et populations de référence}

\section{a) Qualité de l'échantillonnage}

Le choix des animaux de la population de référence n'est pas anodin. Il est d'abord nécessaire de couvrir une variabilité génétique suffisamment large pour garantir que les modèles statistiques appliqués sont en mesure de détecter les marqueurs liés à des variations du phénotype. Chez les bovins laitiers, l'optimum est de disposer de séries de testage (sur descendance) entières « de façon à ce que les " bons 》 comme les « mauvais 》 soient bien représentés » (RobertGranier et al 2011). Ensuite, la population de référence doit être suffisamment apparentée à la population des candidats. Les évaluations génomiques actuelles sont d'autant plus précises que l'apparentement entre ces deux populations est élevé (Legarra et al 2008).

\section{b) De taille " suffisamment grande »}

Les applications actuelles de la sélection génomique sont pour l'essentiel issues d'une nouvelle valorisation des dispositifs usuels de collecte de données (contrôle laitier sur des milliers d'animaux, testage sur descendance) et d'évaluation génétique. La taille des populations de référence actuelles des trois principales races laitières françaises, à savoir Prim'Holstein, Montbéliarde et Normande, est idéale : quelques milliers de taureaux, principaux ascendants des populations actuelles dans les élevages ; issus des dispositifs de testage sur descendance ; disposant de phénotypes précis, leurs index sur descendance étant des pseudo-performances issues des évaluations génétiques classiques portant sur plusieurs millions de performances! 
Figure 3. Taille de la population de référence en fonction de l'héritabilité du caractère et du niveau de Coefficient de Détermination (CD) des index génomiques (d'après Hayes et al 2009).

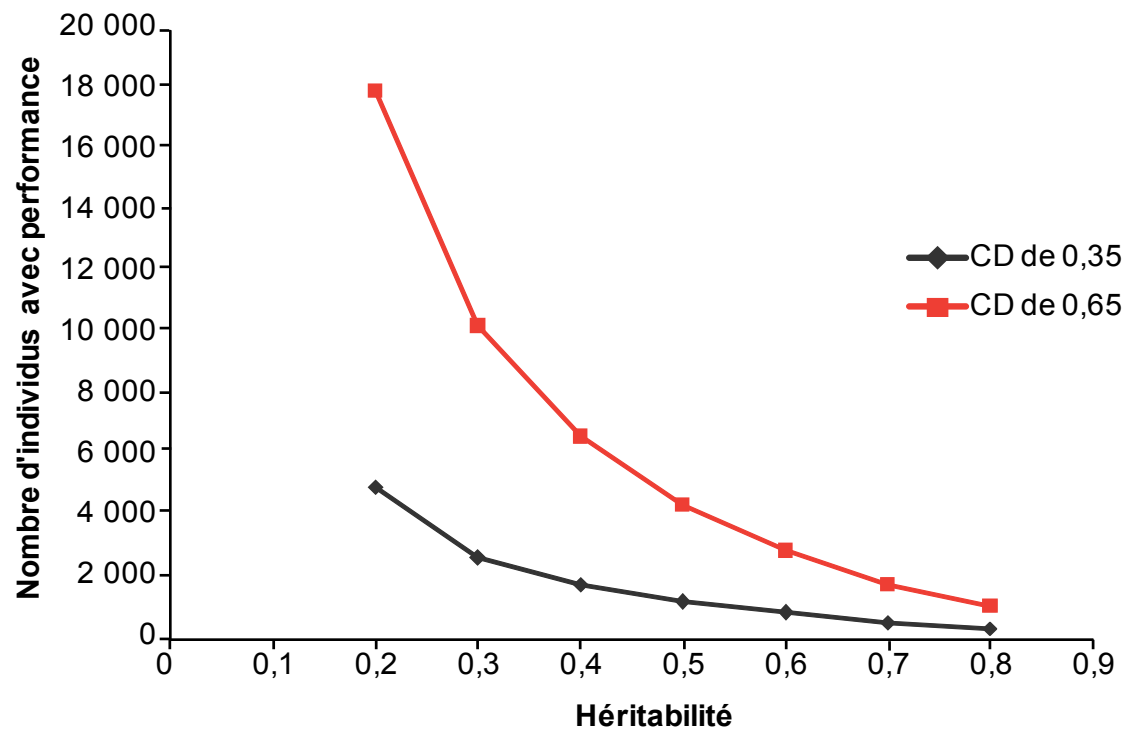

Pour de nouveaux caractères, il ne sera généralement pas possible d'obtenir des données aussi nombreuses ni d'indexer les taureaux sur descendance. Dans ce cas, les populations de référence devront être constituées d'individus avec des performances propres. La figure 3 montre que pour des caractères moyennement héritables (production laitière par exemple) il faut réunir environ 10000 individus avec performances propres pour atteindre un CD de 0,65 (Hayes et al 2009). Les caractères liés à la robustesse ont souvent une héritabilité faible, voire très faible (par exemple entre 1 et $5 \%$ pour la résistance aux mammites cliniques et les caractères de fertilité femelle). Dans ces conditions, plusieurs dizaines de milliers d'animaux seront nécessaires pour nombre de caractères de robustesse. Notons que pour la santé, l'expression du caractère dépend du contexte infectieux : lorsque la prévalence est faible, le nombre d'individus « informatifs » parce que malades peut être très nettement inférieur au nombre d'individus à suivre. Les animaux sains sont moins informatifs, car leur niveau d'exposition au risque est difficile à apprécier. Au final, la taille des populations de référence, et en tout cas des populations à phénotyper, peut être importante, selon les phénotypes et les objectifs que l'on se fixe.

\section{c) Notion d'entretien de la population} de référence

Sonesson et Meuwissen (2009) montrent (figure 4) au travers de simulations que la précision des index génomiques décroît rapidement si la population de référence n'est pas renouvelée ou entretenue. Ce niveau de dégradation est plus ou moins important selon les dispositifs de sélection, le niveau des déséquilibres est un atout important pour assurer l'entretien des populations de référence.

\section{d) Phénotypage?}

Jusqu'à présent, le contrôle de performances était conçu pour être appliqué à une population suffisante pour couvrir l'ensemble des besoins de sélection. A l'avenir, plusieurs types de phénotypages sont à envisager. Le contrôle de performances tel qu'il existe actuellement doit rester à un niveau assez élevé (permettre l'obtention d'un index sur descendance avec un $\mathrm{CD}$ supérieur à 0,70 pour une majorité des taureaux) pour garantir la bonne précision des index pour les caractères essentiels, contrôler leur validité et mesurer leur efficacité, limiter les biais qui pourraient être générés par un phénotypage dans des milieux trop différents des conditions d'élevage moyennes. Mais l'extension à de nouveaux caractères ne sera pas possible à la même échelle et on devra accepter des dispositifs à plusieurs niveaux. Pour ces nouveaux caractères (par exemple la composition fine du lait avec le programme PhénoFinlait), se développent actuellement des initiatives, dites de phénotypage et génotypage, caractérisées par i) une approche multidisciplinaire pour comprendre et identifier avec précision le phénotype d'intérêt ; ii) un suivi le plus précis possible des individus ou collatéraux d'une population de référence ; iii) l'enregistrement de l'environnement, c'est-à-dire des conditions dans lesquelles le phénotype a été observé et $i v)$ le génotypage des animaux, (programme PhénoFinlait, Esvan et al

Figure 4. Evolution du Coefficient de Détermination (CD) des index génomiques selon le niveau d'entretien de la population de référence (d'après Sonesson et Meuwissen 2009).

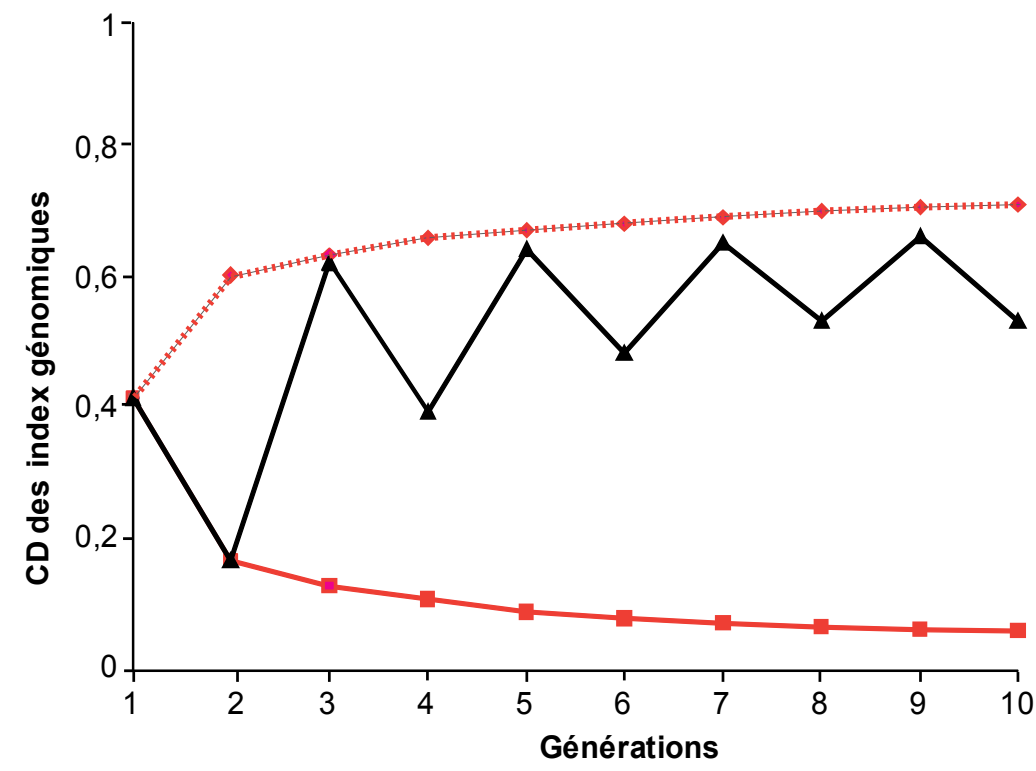

3 scénarios d'entretien de la population de référence (3000 individus)

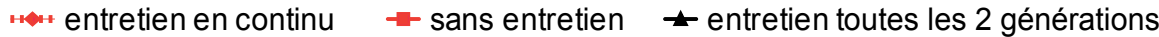


2010). On passe donc à une approche plus intégrée : le phénotype est l'expression d'un génotype dans un environnement donné.

Pour de nombreux nouveaux caractères, cette évolution de la collecte de phénotypes (par rapport à la combinaison contrôle de performances et testage sur descendance), pose la question du passage de populations de référence mâles évaluées sur descendance à des populations de référence femelles (ou mâles) évaluées sur performances propres, avec nettement moins de précision.

\section{2 / Entre collaboration et com- pétition, un paysage qui change}

a) Partenariats entre recherche et développement

Les programmes de phénotypage reposent actuellement sur des partenariats de Recherche et Développement (R\&D) entre recherche publique (INRA, ANR), instituts techniques et partenaires privés (Apis-Gène, Cniel, Entreprises de Sélection (ES), Organismes de Contrôle Laitier devenus Entreprises de Conseil en Elevage...). Ceci a plusieurs raisons, en particulier i) la complexité croissante des caractères d'intérêt, notamment ceux liés à la robustesse des animaux (résistance aux maladies, par exemple) ou à la qualité des produits pour les consommateurs finaux; ii) l'évolution extrêmement rapide des techniques et des connaissances; iii) les besoins d'approche intégrée (caractérisation du milieu); iv) l'importance des niveaux d'investissement financiers.

Le domaine de la composition fine du lait est assez illustratif du développement de ces collaborations : aux PaysBas (Visker et al 2006), en France avec PhénoFinlait (www.phenofinlait.fr), au Danemark et en Suède (www.milkgenomics.dk).

\section{b) Consortiums et partenariats interna- tionaux}

Dans l'objectif de mutualiser les efforts en vue de la constitution de populations de référence de taille suffisante, le monde de la génétique a vu ces dernières années l'apparition de consortiums - NordAméricain (Muir et al 2010), Eurogenomics (David et al 2010) - ou de collaborations telles qu'Intergenomics (Jorjani et al 2010). Pour la race Holstein et la France, le consortium Eurogenomics correspond à la collaboration des entreprises de sélection et des centres de recherche allemands, français, néerlandais, nordiques, espagnols et polonais. Cette collaboration a conduit à une population de référence globale plus de quatre fois plus grande que la seule population française et comparable à celle des Nord-Américains.

c) A l'heure de la génomique, le phénotype est roi!

A l'échelle nationale, les phénotypes et les mesures qui permettent de les apprécier sont devenus le véritable facteur limitant, et donc un des enjeux majeurs pour demain. Différentes stratégies sont mises en œuvre. D'une part, disposer d'un phénotype que le concurrent n'a pas peut être vu comme un avantage compétitif qu'on ne souhaite pas partager. Cependant, on peut supposer que le concurrent ne restera pas longtemps sans réagir, de sorte que des bases analogues et séparées vont coexister. Une mise en commun, même entre compétiteurs, est alors la meilleure solution pour maximiser l'efficacité du dispositif et réduire les coûts. Ceci explique le paradoxe que l'on observe actuellement, avec un mouvement de privatisation des données au niveau local, notamment au travers de collaborations, voire demain de fusions entre acteurs traditionnels de la collecte (entreprises de conseil en élevage) et de la sélection (entreprises de sélection, voire organismes de sélection). Et dans le même temps s'instaure un partage entre acteurs qui avaient peu de relations auparavant, comme dans Eurogenomics.

Notons également l'ouverture ou l'émergence de nouveaux acteurs. Ainsi en 2012, GDS France (Fédération des groupements de défense sanitaire) a conclu avec France Génétique Elevage un partenariat dont l'objet premier est de promouvoir et renforcer les collaborations nécessaires aux développements d'évaluations génomiques dans le domaine de la santé animale. Autre exemple : les discussions et les collaborations s'établissent entre les industriels, fabricants d'instruments (robots de traite, capteurs...) et les acteurs traditionnels de la génétique.

\section{Conclusion}

Le progrès génétique sur la production laitière, important pendant plusieurs décennies, a eu des répercussions indirectes négatives sur la robustesse des animaux (baisse de la fertilité femelle, augmentation de la sensibilité aux infections mammaires...). Bien que ces caractères aient été introduits progressivement dans les objectifs de sélection à partir de la fin des années 1990 et en particulier avec la réforme de l'ISU en 2001, l'amélioration du niveau génétique reste faible pour ces caractères. Le déploiement de la sélection génomique dans les trois principales races laitières françaises depuis 2009 permet d'envisager une bien meilleure efficacité de la sélection sur les caractères fonctionnels du fait d'une connaissance du niveau génétique des reproducteurs, mâles et femelles, en amont des choix de sélection. Par ailleurs, la possibilité de doublement du progrès génétique a conduit à réformer l'ISU, visant, à progrès génétique constant sur les caractères de production laitière, l'augmentation du poids des caractères fonctionnels. Ainsi, le poids global accordé aux qualités d'élevage est passé de 37 à 50\% en 2012 en race Prim'Holstein. Les principes de la sélection génomique et en particulier le fait de dissocier la phase de collecte de données de la phase d'indexation, associés à l'évolution rapide des méthodes de mesure (spectres MIR, capteurs en élevage, imagerie...), sont à l'origine de nombreux programmes de phénotypage pour évaluer de nouveaux caractères. Les initiatives sont nombreuses dans le domaine de la santé animale : l'enquête menée en 2010 par France Génétique Elevage en collaboration avec le Cniel a conclu que ce champ était prioritaire parmi les souhaits des éleveurs et de leurs filières. Les développements sur l'efficacité et les rejets, notamment de méthane, sont également une perspective réelle mais complexe et qui nécessiteront des collaborations probablement à l'échelle nationale et internationale. Enfin, déjà bien engagés au travers du programme PhénoFinlait, les travaux sur les caractères de composition fine du lait pour aborder des aspects de qualité nutritionnelle des produits sont un enjeu de filière majeur qui devrait déboucher rapidement sur des évaluations génomiques.

A l'ère de la génomique, finalement le phénotype est roi! Plus précisément, l'accès à des mesures de phénotype et la construction de bases de données de références est devenu un champ de compétition forte. Des évolutions importantes de l'organisation des dispositifs génétiques - nouveaux acteurs, collaborations et fusions entre acteurs, évolution des collaborations entre la recherche et les acteurs de développement sont en cours, avec leur lot de risques et d'opportunités. Plus qu'hier, la mutualisation et le partage sont indispensables pour faire face aux coûts et à la complexité des dispositifs de collecte de nouveaux caractères.

Néanmoins, si des outils efficaces sont disponibles ou vont l'être, l'amélioration de la durabilité des animaux ou de ses composantes n'est pas automatiquement garantie. Tout d'abord, le processus de sélection (chez les ruminants) s'appuie sur un consensus sur les orientations globales et les pondérations entre les caractères à l'échelle d'une popula- 
tion. C'est là une limite à la différenciation ou segmentation que certains acteurs pourraient viser. Par ailleurs, pour qu'un caractère soit pris en compte efficacement dans des choix de sélection, il faut que l'éleveur ait un intérêt économique au niveau de son élevage, soit par réduction des charges, soit par augmentation ou maintien de ses produits. Cet intérêt économique n'est pas forcément simple à définir (par exemple pour l'impact sur l'environnement), et est dépendant du système d'élevage, ou bien encore peut être l'objet de négociations complexes au sein des filières (quel profil en acide gras demain ? quelle traduction dans les grilles de paiement du lait ?). Le programme OSIRIS (soutenu par le Ministère chargé de l'agriculture, sur fonds CASDAR dédiés au Développement agricole et rural) a ainsi été mis en place pour aborder la question de l'intégration de ces nouveaux caractères dans les objectifs de sélection.
Par ailleurs, la sélection génomique n'est disponible en France pour l'instant que dans les trois principales races. Un enjeu majeur à court terme réside dans la mise en place de la sélection génomique pour les autres races, malgré leurs effectifs modérés et les moindres capacités d'investissement des entreprises et organismes chargés de leur sélection. Un programme de recherche (GEMBAL, soutenu par l'ANR et ApisGène) est en cours pour assurer cette extension à d'autres races.

Enfin, quand on traite de durabilité, la variabilité génétique dans la population d'animaux est un autre paramètre essentiel pour garantir les capacités de réponse à la sélection et les possibilités d'évolution de l'orientation des races. La sélection génomique, en réduisant fortement l'investissement à l'échelle du taureau candidat peut faciliter l'ouverture des origines, l'augmentation du nombre de reproducteurs disponibles et leur remplacement rapide. Les données moléculaires peuvent être une nouvelle source pour apprécier et suivre la consanguinité et l'apparentement. Colleau et al (2009) ont montré qu'il était possible de ralentir le rythme d'augmentation de la consanguinité moyenne tout en doublant le progrès génétique annuel. Mais tout est une question de choix, et ce n'est qu'à la condition que le « star system » soit abandonné au profit de l'utilisation d'un nombre plus important de taureaux chacun utilisés sans excès, que l'on préservera la variabilité génétique mieux que ce que l'on ne pouvait le faire auparavant.

\section{Remerciements}

Les auteurs remercient Pascale Le Mézec et Etienne Verrier pour leur relecture critique d'un premier manuscrit.

\section{Références}

Agerholm J.S., 2007. Inherited Disorders in Danish Cattle. Acta Pathologica, Acta Pathol. Microbiol. Immunol. Scand., 115, s122, 1-76.

Banos G., 2010. Past, present and future of international genetic evaluations of dairy bulls. In: $9^{\text {th }}$ WCGALP, Leipzig, Germany, paper 33.

Bewley J.M., Peacock A.M., Lewis O., Boyce R.E., Roberts D.J., Coffey M.P., Kenyon S.J., Schutz M.M., 2008. Potential for estimation of body condition scores in dairy cattle from digital images. J. Dairy Sci., 91, 3439-3453.

Bodin L., Bolet G., Garcia M., Harreau H., Larzul C., David I., 2010. Robustesse et canalisation: vision de généticiens. In : Numéro spécial, Robustesse, rusticité, flexibilité, plasticité, résilience... Sauvant D., Perez J.M. (Eds). INRA Prod. Anim., 23, 11-22.

Boichard D., Barbat A., Briend M., 1998. Evaluation génétique des caractères de fertilité femelle chez les bovins laitiers. Renc. Rech. Rum., 103-106.

Boichard D., Chung H., Dassonneville R., David X., Eggen A., Fritz S., Gietzen K.J., Hayes B.J., Lawley C.T., Sonstegard T.S., Van Tassell C.P., VanRaden P.M., Viaud K., Wiggans G.R., 2012. Design of a bovine lowdensity SNP array optimized for imputation. Plos One. 7, e34130.

Bonaïti B., Moureaux S., Mattalia S., 2005. Bilan et paramètres génétiques des mammites cliniques collectées par le contrôle laitier dans les races Montbéliarde, Normande et Prim' Holstein. Renc. Rech. Rum., 271-274.

Cecchinato A., De Marchi M., Gallo L., Bittante G., Carnier P., 2009. Mid-infrared spectroscopy predictions as indicator traits in breeding programs for enhanced coagulation properties of milk. J. Dairy Sci., 92, 5304-5313.

Chilliard Y., Martin C., Rouel J., Doreau M., 2009. Milk fatty acids in dairy cows fed whole crude linseed, extruded linseed, or linseed oil, and their relationship with methane output. J. Dairy Sci., 92, 5199-5211.
Cniel, 2011. Lait, produits laitiers et société : France 2025. Paris, France, p20.

Colleau J.J., Régaldo D., 2001. Définition de l'objectif de sélection dans les races bovines laitières. Renc. Rech. Rum., 329-332.

Colleau J.J., Fritz S., Guillaume F., Baur A., Dupassieux D., Boscher M.Y., Journaux L., Eggen A., Boichard D., 2009. Simulation des potentialités de la sélection génomique chez les bovins laitiers. Renc. Rech. Rum., 419.

Cramer G., Lissemore K.D., Guard C.L., Leslie K.E., Kelton D.F., 2008. Herd- and cowlevel prevalence of foot lesions in Ontario dairy cattle. J. Dairy Sci., 91, 3888-3895.

Dal Zotto R., De Marchi M., Cecchinato A., Penasa M., Cassandro M., Carnier P., Gallo L., Bittante G., 2008. Reproducibility and repeatability of measures of milk coagulation properties and predictive ability of mid-infrared reflectance spectroscopy. J. Dairy Sci., 91, 4103-4112.

Danchin-Burge C., Leroy G., Moureaux S., Verrier E., 2012. Evolution of the genetic variability of 8 French dairy cattle breeds assessed by pedigree analysis. J. Anim. Breed. Gen. 129, 206-217.

Dassonneville R., Fritz S., Ducrocq V., Boichard D., 2012. Short communication: Imputation performances of three low density marker panels in beef and dairy cattle. J. Dairy Sci., 95, 4136-4140.

David X., de Vries A., Feddersen E., Borchersen S., 2010. International Genomic Cooperation - EuroGenomics significantly improves reliability of Genomic evaluations. Interbull Bul. 41, 77-78.

De Marchi M., Fagan C.C., O’Donnell C.P., Cecchinato A., Dal Zotto R., Cassandro M., Penasaand M., Bittante G., 2009. Prediction of coagulation properties, titratable acidity, and $\mathrm{pH}$ of bovine milk using mid-infrared spectroscopy. J. Dairy Sci., 92, 423-432.
Dezetter C., Le Mézec P., 2011. Bilan d'indexation des races laitières : résultats de la campagne 2010, Institut de l'Elevage CR $\mathrm{n}^{\circ} 001172023$, http://idele.fr/domaines-techniques/ameliorer-le-troupeau/indexresultats/publication/idelesolr/recommends/bil an-dindexation-des-races-bovines-laitieres2010-bil.html

Douguet M., Brochard M., Mattalia S., 2008. Enregistrement des mammites cliniques dans le SIG : Etat des lieux de la remontée et degré d'exhaustivité, Institut de 1'Elevage CR $\mathrm{n}^{\circ} 010872025$.

Ducrocq V., 2005. An improved model for the French genetic evaluation of dairy bulls on length of productive life of their daughters. J. Anim. Sci., 80, 249-256.

Ducrocq V., 2006. Current status and prospects of survival analysis in animal breeding. In: $8^{\text {th }}$ WCGALP, Belo Horizonte, Brasil, paper 26-07.

Ducrocq V., Boichard D., Barbat A., Larroque H., 2001. Intégration des caractères fonctionnels dans un index de synthèse pour les races bovines laitières : de la théorie à la pratique. Renc. Rech. Rum., 333-336.

Egger-Danner C., Obritzhauser W., FuerstWaltl B., Grassauer B., Janacek R., Schallerl F., Litzllachner C., Koeck A., Mayerhofer M., Miesenberger M., Schoder G., Sturmlechner F., Wagner A., Zottl K., 2010. Registration of health traits in Austria: Experience review. In: $37^{\text {th }}$ ICAR Mtg., Riga, Latvia, 69-78.

Eriksson J.A., 2006. Swedish sire evaluation of hoof diseases based on hoof trimming records. Interbull Bull. 35, 49-52.

Esvan S., Dragan C., Varenne A., Astruc J.M., Barillet F., Boichard D., Brunschwig P., Dubrulle A., Faucon-Lahalle F., Ferlay A., Lagrifoul G., Larroque H., Legarto J., Palhiere I., Peyraud J.L., Rupp R., Brochard M., 2010. PhénoFinlait, $1^{\text {ers }}$ résultats : influence de l'alimentation, de l'état physiologique et de la génétique sur la composition en acides gras 
des laits de vache, brebis et chèvre. Renc. Rech. Rum., 385-388.

Ferrand M., Huquet B., Barbey S., Barillet F., Faucon F., Larroque H., Leray O., Trommenschlager J.M., Brochard M., 2011. Determination of fatty acid profile in cow's milk using mid-infrared spectrometry: Interest of applying a variable selection by genetic algorithms before a PLS regression. Chem. Int. Lab. Syst., 106, 183-189.

Friggens N.C., Thorup V.M., Edwards D., 2011. Estimation à la ferme du bilan énergétique des vaches laitières à l'aide des mesures fréquentes de poids vif et de note d'état corporel. Renc. Rech. Rum., 39-42.

Fritz S., Guillaume F., Croiseau P., Baur A., Hoze C., Dassonneville R., Boscher M.Y., Journaux L., Boichard D., Ducrocq V., 2010. Mise en place de la sélection génomique dans les trois principales races françaises de bovins laitiers. Renc. Rech. Rum., 455-458.

Gonda M.G., Chang Y.M., Shook G.E., Collins M.T., Kirkpatrick B., 2006. Genetic variation of mycobacterium avium ssp. paratuberculosis infection in US Holsteins. J. Dairy Sci., 89, 1804-1812.

Halachmi I., Polak P., Roberts D.J, Klopcic M., 2008. Cow body shape and automation of condition scoring. J. Dairy Sci., 91, 44444451 .

Hayes B.J., Bowman P.J., Chamberlain A.J, Goddard M.E., 2009. Genomic selection in dairy cattle: progress and challenges. J. Dairy Sci., 92, 433-443.

Hegarty R.S., Goopy J.P., Herd R.M., McCorkell B., 2007. Cattle selected for lower residual feed intake have reduced daily methane production. J. Animal Sci., 85, 1479 1486.

Jorjani H., Jakobsen J.H., Forabosco F., Hjerpe E., Fikse W.F., 2007. An international perspective on breeding for robustness of dairy cattle. In: 58 ${ }^{\text {th }}$ EAAP Mtg., Dublin, Ireland, Book of abstracts, 13-24.

Jorjani H., Zumbach B., Dürr J., Santus E., 2010. Joint genomic evaluation of BSW populations, Interbull Bull., 41, Paris, France, 814.

Larroque H., Ducrocq V., 2001. Relationship between type and longevity in the Holstein breed. Genet. Sel. Evol., 33, 39-59.

Lassen J., Hansen N., Sørensen M.K., Aamand G.P., Christensen L.G., Madsen P., 2003. Genetic relationship between body condition score, dairy character, mastitis and diseases other than mastitis in first-parity Danish Holstein cows. J. Dairy Sci., 86, 3730-3735.

Lassen J., Sørensen M. K., Madsen P., Ducrocq V., 2007. An approximate multitrait model for genetic evaluation in dairy cattle with a robust estimation of genetic trends. Gen. Sel. Evol., 39, 353.

Lassen J., Løvendahl P., Madsen J., 2010. Experiences with large scale individual measurements of methane emission in dairy cattle using a Fourier Transform Infrared (FTIR) measuring unit. In: $9^{\text {th }}$ WCGALP, Leipzig, Germany, paper 247.

Le Mézec P., Barbat-Leterrier A., Barbier S., Gion A., Ponsart C., 2010. Fertilité des principales races laitières - Bilan 1999-2008, Institut de 1'Elevage CR n001072030.

Legarra A., Robert-Granié C., Manfredi E., Elsen J.M., 2008. Performance of genomic selection in mice. In Genetics, 108.088575.

McParland S., Banos G., Wall E., Coffey M.P., Soyeurt H., Veerkamp R.F., Berry D.P., 2011. The use of mid-infrared spectrometry to predict body energy status of Holstein cows. J. Dairy Sci., 94, 3651-3661.

Minozzi G., Buggiotti L., Stella A., Strozzi F., Luini M., Williams J.L., 2010. Genetic loci involved in antibody response to Mycobacterium avium ssp. paratuberculosis in cattle. Plos One, 5, e11117.

Muir B., Van Doormaal B., Kistemaker G., 2010. International genomic cooperation North American perspective, Interbull Bul., 41, 71-76.

Pinares-Patiño C.S., Baumont R., Martin C., 2003. Methane emissions by Charolais cows grazing a monospecific pasture of timothy at four stages of maturity. Can. J. Animal Sci., 83, 769-777.

Pryce J.E., Harris B.L., Bryant J.R., Montgomerie W.A., 2009. Do robust dairy cows already exist?. EAAP Sci., Series 126 , 99-112.

Robert-Granié C., Legarra A., Ducrocq V., 2011. Principes de base de la sélection génomique. In : Numéro spécial, Amélioration génétique. Mulsant P., Bodin L., Coudurier B. Deretz S., Le Roy P., Quillet E., Perez J.M. (Eds). INRA Prod. Anim., 24, 331-340.

Rogers W. G., 2005. US perspective: the importance of functional traits and crossbreeding in dairy cattle. In: $56^{\text {th }}$ EAAP Mtg., Uppsala, Sweden, Book of abstracts, 11, 43.

Rupp R., Boichard D., 1997. Evaluation génétique des bovins laitiers sur les comptages de cellules somatiques pour l'amélioration de la résistance aux mammites. Renc. Rech. Rum., 211-214.

Rupp R., Boichard D., 1999. Genetic parameters for clinical mastitis, somatic cell score, production, udder type traits, and milking ease in first lactation Holsteins. J. Dairy Sci., 82 2198-2204.
Schaeffer L.R., 2006. Strategy for applying genome-wide selection in dairy cattle. J. Anim. Breed. Gen., 123, 218-223.

Sonesson A.K. et Meuwissen T.H.E., 2009. Testing strategies for genomic selection in aquaculture breeding programs. Gen. Sel. Evol. 41: 37 doi:10.1186/1297-9686-41-37.

Soyeurt H., Dardenne P., Dehareng F. Lognay G., Veselko D., Marlier M., Bertozzi C., Mayeres P., Gengler N., 2006. Estimating fatty acid content in cow milk using MidInfrared Spectrometry. J. Dairy Sci., 89, 36903695 .

Soyeurt H., Colinet F.G., Arnould V.M.R., Dardenne P., Bertozzi C., Renaville R., Portetelle D., Gengler N., 2007. Genetic variability of lactoferrin content estimated by mid-infrared Spectrometry in bovine milk. J. Dairy Sci., 90, 4443-4450.

Soyeurt H., Dehareng F., Mayeres P., Bertozzi C., Gengler N., 2009a. Variation of Delta(9)-desaturase activity in dairy cattle. J. Dairy Sci., 91, 3211-3224.

Soyeurt H., Bruwier D., Romnee J.M., Gengler N., Bertozzi C., Veselko D., Dardenne P., 2009b. Potential estimation of major mineral contents in cow milk using midinfrared spectrometry. J. Dairy Sci., 92, 24442454.

Stoop M., De Jong G., Van Pelt M., Van der Linde C., 2010. Implementation of a claw health index in The Netherlands. Interbull Bull., 42, 95-99.

Uggla E., 2008. Genetic correlation between feet and leg type traits and claw health in Swedish dairy cattle. Swedish University of Agricultural Sciences, Uppsala, Suède, 40p.

VanRaden P.M., Olson K.M., Null D.J., Hutchison J.L., 2011. Harmful recessive effects on fertility detected by absence of homozygous haplotypes. J. Dairy Sci., 94, 6153-6161.

Verrier E., Le Mézec P., Boichard D. Mattalia S., 2010. Evolution des objectifs et des méthodes de sélection des bovins laitiers. Bulletin de l'Académie Vétérinaire de France, 163, 73-78.

Veerkamp R., Calus M., de Haas Y., 2012. Selection for feed intake in dairy cattle using genomic selection. $38^{\text {th }}$ ICAR, Cork, Ireland.

Visker M., Heck J., Koks P., LeonKloosterziel K., Meuldijk R., Schennink A., Schopen G., Stoop M., Van Arendonk J., 2006. The Dutch Milk Initiative: genetic improvement of milk quality traits and product innovation. $3^{\text {rd }}$ Int. Symp. Milk Genom. Human Health, Brussels, Belgium. 


\title{
Résumé
}

Alors que l'amélioration génétique a été un des facteurs principaux du développement des filières laitières, elle doit aujourd'hui se renouveler notamment pour davantage contribuer à la durabilité de l'agriculture. Un virage vers une prise en compte des caractères de robustesse des animaux (fertilité, résistance aux mammites, longévité des animaux...) a été pris à la fin des années 1990 mais les résultats sont insuffisants. Aujourd'hui, la sélection génomique est une formidable opportunité que les schémas de sélection saisissent pour augmenter fortement le progrès génétique sur les caractères de robustesse tout en maintenant le progrès constant sur la production laitière. En parallèle nous assistons actuellement à un foisonnement de projets dont le but est de permettre à terme la sélection sur de nouveaux caractères. Les deux premiers domaines concernés sont la santé animale et la composition fine du lait. L'efficacité alimentaire et l'empreinte environnementale sont également une perspective réelle mais complexe, qui nécessitera plus de temps et des collaborations à l'échelle nationale ou internationale. Ces avancées passent par une phase dite de phénotypage (collecte de mesures relatives aux caractères liés au phénotype d'intérêt) plus ou moins complexe et coûteuse. Les dispositifs de phénotypage sont des axes stratégiques majeurs pour les acteurs des schémas de sélection. La révolution génomique induit d'autres changements dans le monde de la sélection, de type organisationnel, avec des réorganisations, des compétitions et des alliances nouvelles entre acteurs historiques (organisations en charge de la mise en œuvre du dispositif génétique français selon la loi sur l'élevage de 1966) ou émergents (entreprises étrangères et/ou d'autres secteurs). La génétique est une carte essentielle à jouer pour atteindre les objectifs de durabilité des élevages et filières de demain.

\begin{abstract}
Genetic selection for sustainable cows and sustainable milk production: genetic basis and opportunities offered by genomic selection

Genetic improvement, being one of the major development factors of the dairy industry, now needs to evolve, in particular to contribute more to agriculture sustainability. A change in direction towards a better awareness of fitness traits (fertility, resistance to mastitis, longevity...) took place at the end of the nineties but its outcome is still limited. Genomic selection offers a remarkable opportunity for selection schemes to strongly increase genetic trends for fitness traits while maintaining constant genetic gain on production. Concurrently, a proliferation of new projects can be observed, with the objective to include new traits in selection programs. The first two fields concerned are animal health and fine milk composition. Feed efficiency and environmental footprints such as methane emission also have a good prospect but are complex to deal with. They will require more time as well as national and international collaborations. These developments involve a complex and costly phenotyping phase (data collection of traits related to the phenotype of interest). These phenotyping projects are strategic issues for selection scheme stakeholders. The genomic revolution induces other organisational changes in the breeding world with restructurings, competitions and new alliances between historical and new players. Genetics is a crucial asset in reaching a true sustainability of dairy herds and the dairy industry in the near future.
\end{abstract}

BROCHARD M., BOICHARD D., DUCROCQ V., FRITZ S., 2013. La sélection pour des vaches et une production laitière plus durables : acquis de la génétique et opportunités offertes par la selection génomique. In : Numéro spécial, La vache et le lait. Faverdin P., Leroux C., Baumont R. (Eds). INRA Prod. Anim., 26, 2, 145-156. 